\title{
Computer Surgery 3D Simulations for a New Teaching-Learning Model
}

\author{
Gonzalo Esteban, Camino Fernandez, Vicente Matellan \\ Dept. of Mechanics, Computer Science and Aeronautics \\ University of Leon, ULE \\ Leon, Spain \\ Email: gestec00@estudiantes.unileon.es \\ camino.fernandez@unileon.es \\ vicente.matellan@unileon.es
}

\author{
Jose Manuel Gonzalo \\ Dept. of Veterinary Medicine, Surgery and Anatomy \\ University of Leon, ULE \\ Leon, Spain \\ Email: jm.gonzalo.orden@unileon.es
}

\begin{abstract}
Using 3D computer simulations for training surgeons is not new. Using e-learning for improving students knowledge acquisition is not new. What we propose is to use 3D computer simulations in such a versatile way that those simulations could act as learning objects designed directly by those who own the experience we want to be transmitted.

In order to achieve this goal, it is necessary to create a model in charge of communications between the learning objects and the simulation. This model ensures that, on the one hand, the simulation offers an interface to the learning process stable enough not to be affected by every small change. On the other hand, the model also ensures that the simulation offers an interface complete enough for adopting any change in the learning process.

The key to solve this contradiction is to take the behavior of the simulation objects out of their control leaving in them just their very basic behavior. This paper presents the problem and the design proposed to solve it in a more detailed way.

Index Terms-computer simulation; e-learning; haptics; surgery;
\end{abstract}

\section{INTRODUCTION AND BACKGROUND}

Nowadays, in the medicine field and more specifically in the context of medical practice, there is a constant process to upgrade and reform the methodology used. Any type of surgery (general, abdominal, ocular, ...) is an example of a procedure that requires for the surgeon to have great skill and precision, which implies a solid groundwork during his formation stage.

Computer-assisted learning refers to the use of computers for educational purposes. This can cover from small multimedia products to complex interactive courses. Information technology and communication are present today in mostly every scope of daily life. Its integration into the teachinglearning processes is a need: by one part, it is necessary to train students to acquire the basics skills in their use, and by the other hand it is desirable to exploit the advantages of its use as a support in the educational process.

However, as technology has progressed, different tools and techniques have added additional value to the training process: practice on animals, cadavers, mannequins, .... Therefore, the interactions during the training stages are difficult because, for example, the tissues of a corpse are not similar to those of a living human being, and consequently lacks of the same physiological behavior, to which must be added that the practice can only be make only once. Although mannequins have many disadvantages, they are the most used because they offer a greater anatomic versatility.

An alternative front which is getting more importance is computer simulation, which opens the door to the application of expert systems. Simulators enable a student to practice with virtual patients, allowing to test their skills in critical situations and provide feedback to monitor throughout all the procedure. Such feedback can be used to refine the skills of the students during the surgery in a way that it can improve the level of efficiency of the student before operating with real subjects. Besides this, the simulator provides students with the opportunity to practice with complex or rare cases, which could be a good way to focus on key situations in the surgery, that would otherwise be ignored until it is faced in real life.

Between the sensory channels of living beings, in the field of simulation we highlight two in particular: sight and touch. The visual component is given by two or three-dimensional computer generated graphics that can faithfully reproduce real world objects. The evolution of this technology has allowed and increased the depth and realism of these graphics; first using monocular images (adding light and shadow, relative size, perspectives projections, ...) and later by a stereo pair (generating an image per eye). Nowadays the technology of the stereo pair has a higher reputation thanks to the introduction of stereoscopic vision devices (monitors, projectors, glasses, ...) as they provide a new level of realism to the vision and that is shown on the vast variety of existing applications (scientific visualization, cinema, video games, ...).

By the other hand, the sense of touch (through haptic technology) is less developed than the vision technologies. In fact, haptic technology requires a bidirectional input / output, which difficults the modelling process because it takes into account a large number of variables. Haptic technology can be divided in two main categories: tactile feedback, which is achieved by touching a surface, allowing to know if is smooth or frictional, hot or cold; and force feedback also known as haptic feedback, which occurs when the user opposes the 
movement or the rotation, for example the reaction when touching a spring. Both categories can be crucial to simulate a medical procedure.

There is a wide range of commercial haptic devices from different manufacturers (SensAble Technologies, ForceDimensions, Novint, ...), although these can be made by research groups for specific projects. Most of these devices incorporate a single component of haptic feedback. Virtual reality is a technological system that can introduce the humans into a recreation of the reality, in such way that he feels part of it having the chance to interact with the environment. The most widely accepted definition is given by Burdea [3] who defined virtual reality by its functionality. To Burdea, virtual reality can recreate worlds or realities in a realistic way, at least in part, which lead to a feeling of immersion. The second feature is virtual reality which Burdea says is that the images used by the system are generated by computer graphics. This is one of the reasons for the delayed explosion of virtual reality, because until not long ago, it was unthinkable with the existing technology to generate real-time graphics to recreate the feeling of immersion. Last feature is that the resulting system has to be interactive, and for that, the generated world should not be static, and on the other hand, has to respond to user actions.

\section{StATE OF THE ART}

The state-of-the-art in research on simulations using haptic devices has many open fronts as it is a subject with a high level of interest due to its wide range of applications. One of the most important fronts is applied medicine and more specifically the field of surgery, in which each research group focuses on a particular type of speciality or in certain medical tests.

The procedure of palpation (when a doctor presses his fingers on an specific area in the patient's skin, searching for signs under it) is very interesting because it requires a multifinger haptic feedback, which is a challenge for a simulation. Although it is in an early stage (due to a lack of commercial haptic devices), there are several research lines in this field using diverse haptic devices like one with a glove shape [5] or any standard haptic device [6]. An example of this type of procedure is a simulator for palpating breasts [7] developed by the University of Gifu using haptic interaction algorithms on certain points and a custom haptic device called HIRO.

Another procedure is the needle puncture, which is very easy to simulate with any haptic device. There are several simulators, such as a trainer for catheter insertion [8] developed by the Advance Studies Centre (Italy) which uses a combination of an stereoscopic vision system and a haptic device to simulate tissues with viscoelastic models. Another interesting line of research is the simulation of a needle to stitch wounds like the simulator in [9], which uses a stereoscopic vision system with a custom haptic device.

Laparoscopy (a surgical process made through small incisions to perform surgery inside a body), which has the most advanced line of research with a higher number of training simulators compared to other research areas. An example of this is the VirtaMed HystSim [10] that, through a modified haptic device provides a learning and training system or the Haptica ProMIS [11] which offers a fairly realistic augmented reality system.

Although there are more examples of the application of this kind of system, it is just necessary to look at some cited examples to realize that medical simulation is an area that offers a large to research projects, because those applications are helped in many aspects (economic, material, educational, ...).

\section{DEFINITION OF THE PROPOSED MODEL}

This research project aims to define a model for the creation of systems to be reconstructed from the different elements that compose a surgical procedure (anatomy, tissues, reactions to stimuli, ...).

Besides the interaction with the user, the model also defines a learning system that reflects the expert knowledge related to each type of surgery. The model defines the way that the interaction system is related to the learning system in such a way that each of the learning units have associated the corresponding elements in the simulation, and that each element of the simulation is able to provide information and results in the learning process. Thus, not only will be possible to incorporate the initial expertise, but will ease the task of incorporate new knowledge from learning experiences.

The learning system will be built of several learning units, each of them consisting of three stages (see figure 1):

1) At the first stage, the teacher, who is an expert in his area, brings all his knowledge to develop a teaching plan that will be used by the students for learning.

2) The second stage is critical because it contains the process of developing the teaching plan. Each unit can be translated as a flowchart, which describes each of the processes to follow during the learning stage. This flow chart is very important because it defines the core of the learning unit.

The information related to each process is determined by an initial knowledge base, composed of both, the combination of theoretical bases in the field and personal experience of the expert in the area. This knowledge base is submitted at all times to a process of constant improvement, because the teacher can change it depending on his needs, previous sessions or new contributions, thus providing important feedback to the system.

3) The last stage is the actual learning process by the students. Here each student will use the created virtual system, in which will be loaded the learning unit developed by the expert, and start the procedure.

At this stage, the student will be submitted to each of the processes that compose the learning unit and will be evaluated in each. For this, the system will indicate the success or failure that is being committed, providing a visual aid for both the student and the teacher. 


\section{Learning Unit}

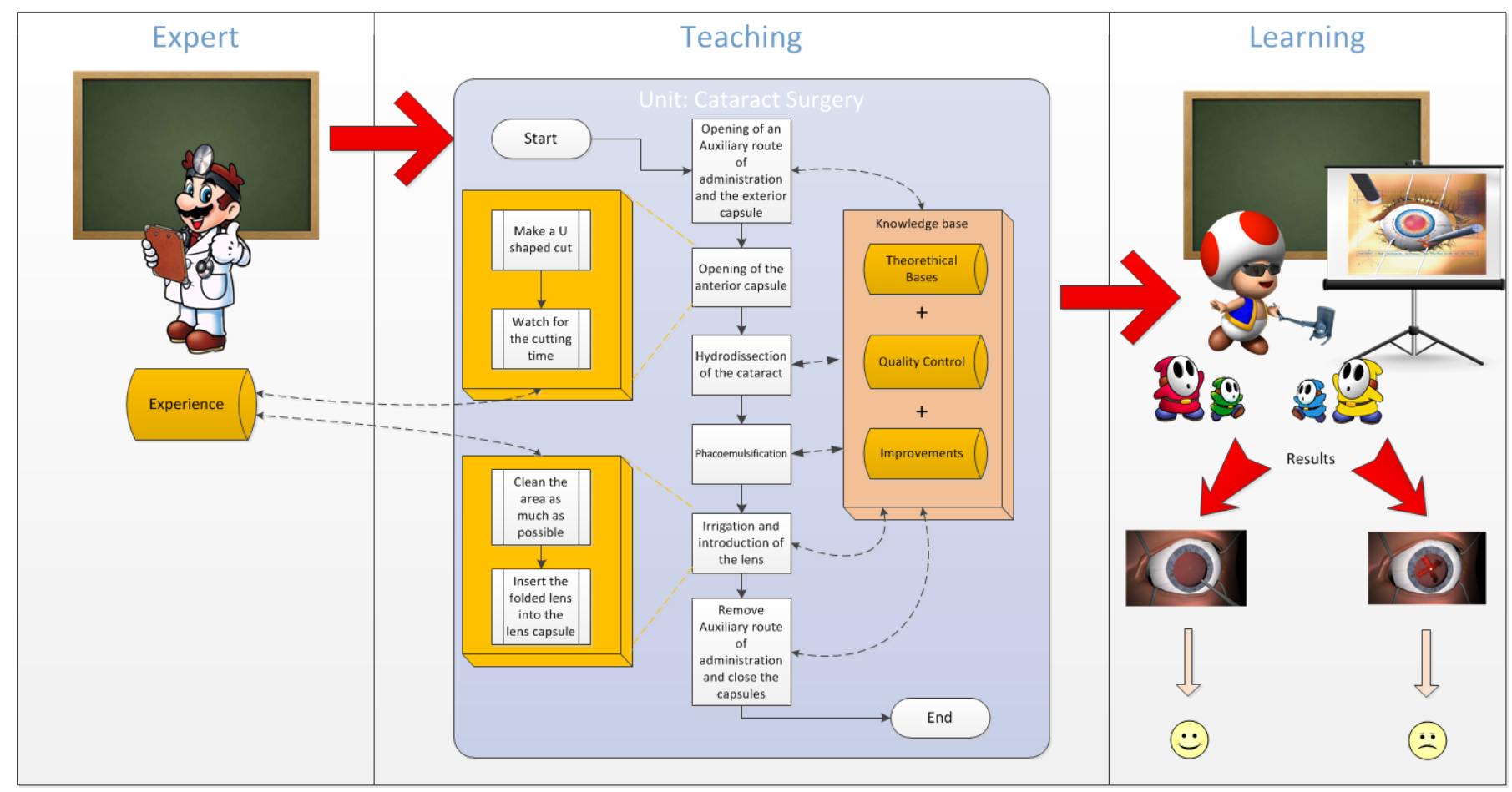

Fig. 1. An example illustrating the components of the model.

At the end of the unit, the teacher will evaluate whether the student has successfully completed or failed the test, allowing to apply feedback to the system, and modifying wherever is necessary in the learning unit for future sessions.

\section{Structure of the Proposed Model}

Following the structure of the proposed model, in a particular learning unit, the main actors involved are: the learning objects and the simulation. Inside learning objects, experience should be expressed, and inside the simulation, real world should be represented. Between them, the model has to provide communication in both directions. From learning objects to the simulation, there must exist a way to express specific tasks as, for instance, "Make a U-shaped cut". From the simulation to the learning objects, there must exist a way to send the results obtained after performing that specific task back to the learning objects for them to evaluate those results.

These two actors have different characteristics that lay the foundations of the middle-layer that has to be defined.

\section{A. Characteristics of the learning objects}

The learning objects reflect the experience to be transmitted. These objects are different depending on the designer. Every designer has an experience and a way of transmitting it that usually differs from the one of another designer. Learning objects can be considered as the dynamic part of the model as long as they change depending on their designer.
For instance, in the example shown in figure 1, an expert can make a U-shaped cut, but another expert may prefer a $\mathrm{C}$ shaped cut. Both have to be able to express it and to evaluate the cuts made by learners.

\section{B. Characteristics of the simulation}

The simulation reflects real world. It includes objects that have real appearance and real behaviour trying to be as much acute as possible. The scene can be considered as the static part of the model as long as it does not change depending on the experience trying to be transmitted. It should always act and react the same way to the same manipulations.

For instance, in the example shown in figure 1, the simulated eye has to behave the same way if the cut is U-shaped or Cshaped. For the simulation there is no difference because the difference only affects the interpretation of the shape related to the one specified in the task. The role of the simulation is limited to report the shape of the cut for the learning object to evaluate the achievement of the goal initially proposed.

\section{The role of the middle-layer}

The role of the middle-layer of the model (figure 2 is to connect the static part to the dynamic one. This layer should offer a way to translate the requirements of the experience (dynamic part) to the fixed possibilities of the simulation (static part), and the other way round. It also has to be able to 

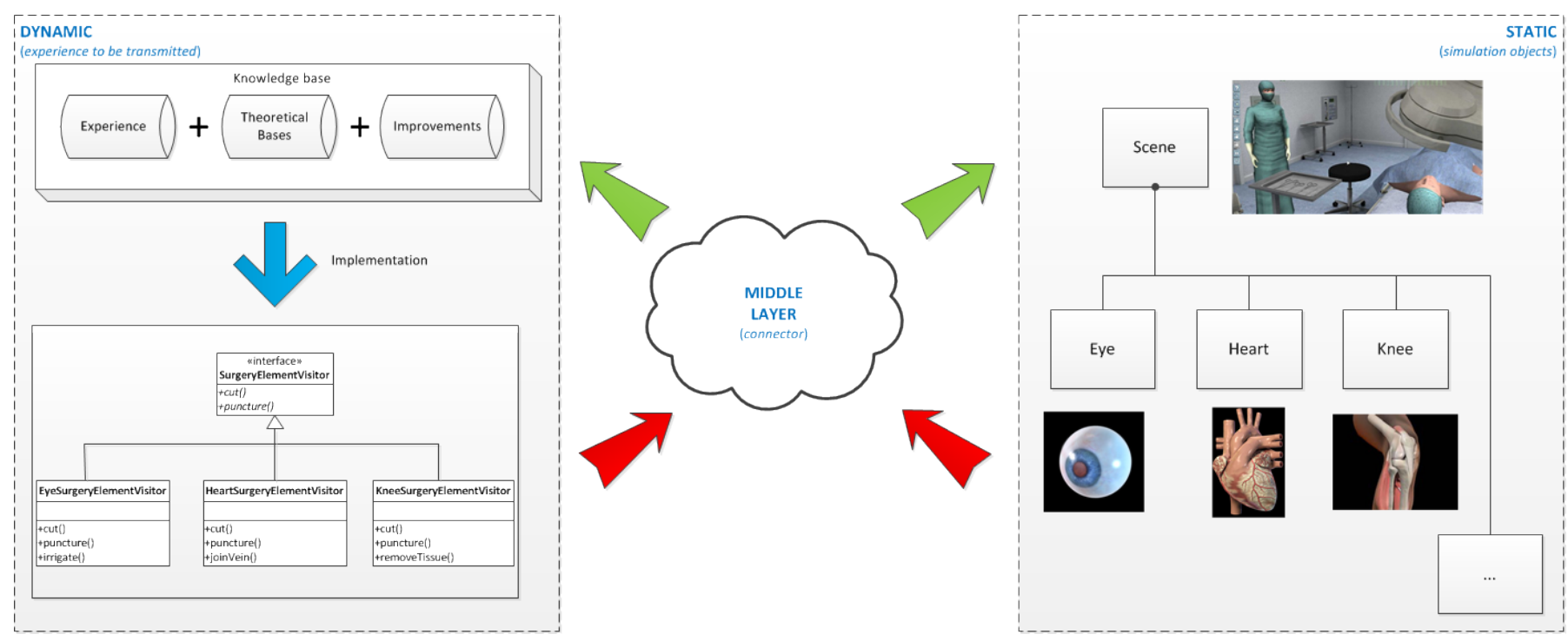

Fig. 2. Structure of the model.

transmit the results obtained in the simulation to the learning objects for them to be interpreted.

In order to provide a stable structure for this situation, the philosophy beneath the visitor design pattern has been used [12]. This pattern represents an operation to be performed on the elements of an object structure and allows to define new operations without changing the classes of the elements on which it operates. This pattern presents many advantages which are useful for this model (such as add new operations, group and ungroup related operations, and break the encapsulation) which will help in the process of constant upgrade of the system.

\section{Summary AND Future RESEARCH}

This short paper defines a model that allow the connection between a virtual reality system and a learning system. Both systems will be developed following the defined model, in order to obtain a simulator capable of be used as a teachinglearning tool.

Once the model is established, the next step will be the development of the necessary learning objects for the cataract surgery and then, the development of the simulation that will be used by that objects. At the same time, the developed software of the middle-layer will act as a connector between both sides to proof the viability, usability and utility of the model.

\section{ACKNOWLEDGEMENTS}

This work has been partially funded by "Programa Profesionales Digitales" (http://www.profesionalesdigitales.es) and a grant from FGULEM (http://www.fgulem.es) in the Research and Technology Innovation Training Program for Graduates. The project includes a laboratory equipped with twelve environments prepared for simulation training -a main frame, two haptic devices and 3D glasses- funded by the project devoted to the impulse of digital contents industry from universities.

\section{REFERENCES}

[1] M. A. Srinivasan and C. Basdogan, Haptics in virtual environments: Taxonomy, research status, and challenges, Computers \& Graphics. vol. 24, issue 4, pp. 393 404, 1997.

[2] T. Coles, D. Meglan and N. W. John, The Role of Haptics in Medical Training Simulators: A Survey of the State-of-the-art, IEEE Transactions on Haptics. vol. 99, 2010.

[3] G. C. Burdea and P. Coiffet, Virtual Reality Technology, WileyBlackwell. 2nd edition, 2003.

[4] R. Webster, R. Haluck, J. Sassani, M. Harris, J. McCaw, C. Billman and J. Gerber, A Joint Research Project in Surgical Simulation with the Penn State University College of Medicine. URL: http://cs.millersville. edu/ $\sim$ webster/haptics/, 2005.

[5] N. A. Langrana, G. Burdea, K. Lange, D. Gomez and S. Deshpande, Dynamic Force Feedback in a virtual Knee Palpation, Artif Intell Med. vol. 6, no. 4, pp. 321 333, 1994.

[6] W. W. Hui Chen, Hanqiu Sun, Pheng-Ann Heng, Dynamic Touch-Enabled Virtual Palpation, Computer Animation and Virtual Worlds. vol. 18, no. 4 5, pp. $339348,2007$.

[7] M. O Alhalabi, V. Daniulaitis, H. Kawasaki and T. Hori, Medical Training Simulator for Palpation of Subsurface Tumor Using Hiro, Eurohaptics 2005. pp. 623 624, 2005.

[8] A. Zorcolo, E. Gobbetti, P. Pili and M. Tuveri, Catheter Insertion Simulation with Combined Visual and Haptic Feedback, in the Proceedings of the First PHANToM Users Research Symposium. Germany, 1999.

[9] R. W. Webster, D. I. Zimmerman, B. J. Mohler, M. G. Melkonian and R. S. Haluck, A Prototype Haptic Suturing Simulator, Stud Health Technol Inform. vol. 81, pp. $567569,2001$.

[10] VirtaMed, HystSim, . URL: http://virtamed.com/cms/home/, 2008.

[11] Haptica, ProMIS Surgical Simulator, . URL: http://www.haptica.com/, 2002.

[12] E. Gamma, R. Helm, R. Johnson and J. M. Vlissides, Design Patterns: Elements of Reusable Object-Oriented Software, Addison-Wesley Professional - 1 edition, 1994 